Conclusion Syphilis proficiency testing in rural facilities carried out by non-laboratory personnel using DTS is feasible. Initial training with on-site monitoring is important to detect any testing problems.

\section{3-S1.06 DIAGNOSTIC ACCURACY OF RAPID POINT-OF-CARE TESTS TO DETECT SYPHILIS: A META-ANALYSES}

doi:10.1136/sextrans-2011-050109.108

${ }^{1} \mathrm{Y}$ Jafari, ${ }^{2} \mathrm{R}$ Peeling, ${ }^{1} \mathrm{~S}$ Shivkumar, ${ }^{3} \mathrm{G}$ Lambert, ${ }^{4} \mathrm{C}$ Claessens, ${ }^{5} \mathrm{~J}$ Cajas, ${ }^{1} \mathrm{M}$ Klein, ${ }^{1} \mathrm{~L}$ Joseph, ${ }^{1} \mathrm{~N}$ Pai. ${ }^{1}$ Mc Gill University, Montreal, Canada; ${ }^{2}$ London School of Hygiene \& Tropical Medicine, UK; ${ }^{3}$ Institut national de santé publique du Québec, Canada; ${ }^{4}$ Laboratoire de santé publique du Québec INSPO, Canada; ${ }^{5}$ Queen's University, Canada

Background The World Health Organization estimates that in 2006, there were 12 million new cases of syphilis. In developing countries, there is a lack of proper screening due to limited laboratory services and long distances from clinics. In developed countries, there is limited access to care among hard-to-reach populations. In this context of disconnect with the health care system, point of care (POC) tests have proven to be an invaluable resource, yet their accuracy needs to be established in order to justify their use.

Method Electronic databases were searched from 1 January 1980 to 24 September 2010 for articles evaluating syphilis POC tests. Data were extracted and a second reviewer independently reviewed a subset of the articles. Subgroups were made according to the index test, the sample tested, and reference standard employed. Pooled sensitivity and specificity were calculated using Hierarchical Summary Receiver Operating Characteristic Curve. Adjustments were made to account for imperfect reference standards.

Results 30 (47\%) from 64 full text articles assessed articles were included in the meta-analysis. The most common kits evaluated were Determine, Bioline, Syphicheck, and Visitect in whole blood and sera samples. Using a Treponema Pallidum (TP) specific reference standard, in sera, the Determine test was the most accurate with a pooled sensitivity of $98.43 \%(96.03,99.94)$ and a specificity of $97.74 \%(96.38,98.92)$. In whole blood, Bioline was the most accurate with a sensitivity of $87.70 \%(84.78,90.58)$ and a specificity of $99.07 \%(98.50,99.59)$. The sensitivity of Determine and Visitect were lower when using whole blood than when using serum. When we adjusted for imperfect reference standards, the pooled parameters of accuracy improved when compared to pooled accuracy under the assumption of a perfect reference standard.

Conclusions Determine with high sensitivity and Bioline with high specificity appeared to perform the best of the tests studied. Higher accuracy in serum warrants the use of serum rather than whole blood wherever possible. Confirmation with non-TP specific reference standard are required to confirm whether the infection is active or treated.

\section{Clinical sciences oral session 2: Genital Human Papillomaviruses \& Trichomoniasis 03-S2.01 LONG-TERM EFFICACY OF HUMAN PAPILLOMAVIRUS VACCINATION AGAINST CIN3 AND INVASIVE CERVICAL CARCINOMA: A REGISTRY BASED PASSIVE FOLLOW-UP OF THE PHASE III TRIAL (PATRICIA)}

doi:10.1136/sextrans-2011-050109.109

\begin{abstract}
${ }^{1} \mathrm{~J}$ Paavonen, ${ }^{2} \mathrm{M}$ Lehtinen, ${ }^{3} \mathrm{M}$ Rana, ${ }^{4} \mathrm{D}$ Apter, ${ }^{5} \mathrm{~T}$ Luostarinen, ${ }^{5} \mathrm{E}$ Pukkala. ${ }^{1} \mathrm{Helsinki}$ University Hospital; ${ }^{2}$ University of Tamepre Tampere, Finland; ${ }^{3}$ Universitu of Tampere, Finland; ${ }^{4}$ Family Federation of Finland, Finland; ${ }^{5}$ Finnish Cancer Registry, Finland
\end{abstract}

Background While phase 3 trials have shown that vaccination against human papillomavirus (HPV) types 16 and 18 prevents persistent HPV type 16 and 18 infections and most high-risk HPV type positive cervical intraepithelial neoplasia (CIN) grade $2+$ lesions, long-term follow-up of the phase 3 cohorts is needed to demonstrate that HPV16/18 vaccination prevents CIN3 and invasive cervical carcinoma (CIN3+).

Methods We used data from the Finnish Cancer Registry for passive follow-up of cluster (age-cohort) and individually randomised cohorts of women born in 1984-1989 to assess incidence rates of CIN3 + in HPV16/18 vaccinated Finnish cohort of the bivalent HPV $16 / 18$ vaccine PATRICIA trial participants $(\mathrm{N}=2404)$ and a reference cohort $(N=7049)$ enrolled from the same communities. Six months after the Phase III trial was closed in 2009 the cohorts were linked with the Finnish Cancer Registry.

Results and Conclusions A pilot study in 2009 showed that the baseline incidence of CIN3 + was 41 per 100000 women years in the reference cohort. Knowing that CIN3+ incidence rapidly increases as the cohorts age, the baseline incidence yields $80 \%$ power to show $70 \%$ vaccine efficacy against CIN3 + in just 5 years. The phase 3 trial included intensive clinical follow-up and thorough health education and counselling which may have modified subsequent risk of cervical neoplasia in all study participants, the incidence rates of CIN3+ need to be validated in a cohort not exposed to any clinical intervention. Preliminary data from such comparison of the incidence rates during passive follow-up the PATRICIA study participants (comprising 50000 women years) and the reference cohort will be reported.

\section{3-S2.02 LONG-TERM EFFICACY OF HUMAN PAPILLOMAVIRUS VACCINATION AGAINST CIN3 AND INVASIVE CARCINOMA: REGISTRY BASED FOLLOW-UP OF A PHASE III TRIAL (FUTURE II)}

doi:10.1136/sextrans-2011-050109.110

${ }^{1} \mathrm{~J}$ Paavonen, ${ }^{2} \mathrm{M}$ Rana, ${ }^{3} \mathrm{D}$ Apter, ${ }^{4} \mathrm{~T}$ Luostarinen, ${ }^{4} \mathrm{E}$ Pukkala, ${ }^{2} \mathrm{M}$ Lehtinen. ${ }^{1} \mathrm{He} /$ sinki University Hospital, Helsinki, Finland; ${ }^{2}$ University of tampere, Finland; ${ }^{3}$ Family Federation of Finland, Finland; ${ }^{4}$ Finnish Cancer Registry, Finland

Background Human papilloma viruses (HPV) 16/18 are known to cause approximately $70 \%$ of cervical cancers. Phase III clinical trials of HPV vaccination have demonstrated $>95 \%$ efficacy against persistent HPV type 16/18 infections and associated cervical intraepithelial neoplasia (CIN) grade $2+$ lesions, and up to $90 \%$ efficacy against all CIN3+ lesions. A long-term follow-up is, however, needed to confirm the protective efficacy against cervical carcinoma. Methods Phase III clinical trial (FUTURE II) consisted of intensive clinical 4-year follow-up including health education and counselling. The intervention potentially affects the incidence of neoplasia also in the placebo group. To increase power of the long-term follow-up and to determine the impact of the clinical intervention as such, a population based reference cohort of similarly aged women not exposed to any intervention was enrolled at the same time from the same communities. The HPV vaccine cohort and placebo vaccine cohort of 16-17-year-old women from the Finnish FUTURE II trial $(\mathrm{N}=1749)$ and a reference cohort of 18-19-year-old women $(N=15744)$ were linked with the Finnish Cancer Registry to determine the incidence of CIN3 and cervical cancer (CIN3+) during the passive follow-up, starting 6 months after the clinical follow-up of the phase III trial was completed.

Results \& Conclusions Currently the incidence of CIN3+ at the age of 20-24 years is 95 per 100000 person years in Finland (http:// www.cancer.fi). The incidence doubles in 5 to 10 years as the cohorts age. Thus, in less than 10 years the cumulative incidence yields $80 \%$ power to demonstrate $90 \%$ vaccine efficacy against cervical CIN3 +. During the first 2 years this passive registry-based follow-up identified no CIN3+ cases in the HPV vaccine cohort, 